\title{
Study of the Parameters Affecting Ion Beam Emerging from Cold Conical Cathode Ion Source
}

\author{
H. El-Khabeary \\ Accelerators \& Ion Sources Department, Basic Nuclear Science Division, \\ Nuclear Research Center, Atomic Energy Authority, P.N.13759, Egypt.
}

(Received on 6 March, 2010)

\begin{abstract}
The aim of this work is study of the parameters affecting ion beam emerging from cold conical cathode ion source. The input discharge and output ion beam characteristics have been measured at different pressures using nitrogen and argon gases. The optimum distance between the ion exit aperture of the cathode and the movable copper ion collector plate has been determined using nitrogen and argon gases. The ion collector plate has been placed at different distances from the ion exit aperture of the cathode equal to 2, 3, 4, 4.5, 5, 5.5 and $6 \mathrm{~cm}$ respectively. It is found that the optimum distance between the ion exit aperture of the cathode and the ion collector plate equals $5 \mathrm{~cm}$ for high output ion beam current. At this optimum distance, the efficiency of the ion source reaches $28.3 \%$ and $21.3 \%$ using nitrogen and argon gases respectively. The divergence angle of the ion beam exit from the cathode aperture has been determined for each distance by measuring the ion beam diameter which obtained on the ion collector plate. It is found that at the optimum distance between the ion exit aperture of the cathode and the ion collector plate, a minimum divergence angle of the ion beam emerging from the ion source equal to $1.14^{0}$ and $2.29^{0}$ using nitrogen and argon gases respectively. Also the aspect ratio of the ion source, the ratio between the radius of the ion exit aperture of the cathode to the distance between the ion exit aperture of the cathode and ion collector plate, has been determined.
\end{abstract}

Keywords: Cold Conical Cathode Ion Source, Divergence Angle, Aspect Ratio

\section{INTRODUCTION}

An ion source is defined as a device in which the gas ions are produced, focused [1], accelerated and emitted as a narrow intense beam [2]. The ion source is the important single element in an ion beam system. The characteristics of the ion source determine to a great extent the performance of the entire system.

In all types of ion sources [3], the ions are produced by an electrical discharge [4] through a gas or vapor of liquid or solid at low pressure. The ionization is produced by electron impact in gaseous discharge. The general requirements are a source of electrons, a small region of relatively high gas pressure, an electric field to accelerate the electrons in order to produce intense gas discharge, plasma, with relatively high electrons and ions density and some mechanism for extracting a collimated parallel high current ion beam. The high pressure discharge region is ended by an electrode, cathode, with small exit hole for output ion beam current. The adjustable parameters of an ion source are electron emission, gas pressure, voltage across the discharge, magnetic field, distance between the electrodes, size of the exit hole, geometry and surface properties of the electrodes and the dimensions of the discharge vessel.

The emerging ion beam from any ion source [5] is divergent due to the aperture effect. However, some emergent ions while going through the aperture hit its sides and are then lost causing the reduction of the ion source efficiency. The divergence in the ion beam is undesirable in many applications because it forces the user to place his specimen very close to the source. This may cause damage to the specimen due to the thermal radiation from the source. In ion thinning of specimens for electron microscopy, a well directed beam of high intensity is required. In particle accelerator [6] applications, it is necessary to use an ion beam with low divergence angle, so that the beam emittance is lower than the accelerator acceptance, otherwise the accelerator tends to reject some part of the beam. Therefore the production of high ion beam current with low divergence angle has many advantages for various applications.

In recent years, there has been a rapid growth in the utilization of ion sources for a variety of applications, including nuclear physics, isotopes separators, multiampere beams for fusion applications and for industrial production applications. Also they are useful tools in ion implantation, ion sputtering [7], ion etching, ion beam machining, ion beam surface modification [8] and neutron generators. As a consequence of this growth, there has been the need for a better understanding of the factors which affect the optical quality [9] of ion beams.

\section{DESCRIPTION OF THE ION SOURCE}

Fig. (1) shows schematic drawing of a cold conical cathode ion source [10] and its associated electrical circuit. It consists of copper anode disc, A, of diameter equals $37 \mathrm{~mm}$ and copper conical cathode, $\mathrm{C}$, of outer diameter equals $37 \mathrm{~mm}$, inner diameter equals $23 \mathrm{~mm}$, length equals $12 \mathrm{~mm}$ and central aperture of $2 \mathrm{~mm}$ diameter. Both the anode and the cathode are isolated with two confinement rings, I, made from perspex insulator for confining the electrical discharge in the central zone between them. The anode and the cathode are placed inside an insulator cylinder made from perspex material of inner diameter equals $4 \mathrm{~cm}$, outer diameter equals $5 \mathrm{~cm}$ and length equals $5 \mathrm{~cm}$. The movable copper collector plate is placed at a distance equal to $4 \mathrm{~cm}$ from the ion exit aperture of the cathode. The working gas is admitted to the ion source through a hole of $1 \mathrm{~mm}$ diameter in the outer surface of the perspex insulator cylinder. The anode is connected to $10 \mathrm{kV}$ positive power supply for initiating the glow discharge between the anode and the cathode. A milli - ampere meter is used to measure the electrical discharge current between the anode and the cathode, while the kilo voltmeter is used to measure the electrical discharge voltage between them. The cathode is connected to earth and the 
collector plate is connected to earth through micro-ampere meter to measure the ion beam current emerge from central aperture of the cathode.

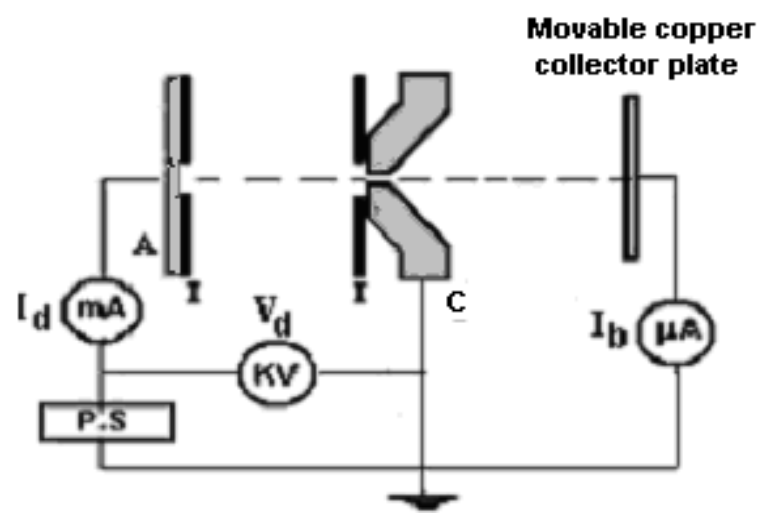

FIG. 1: Cold conical cathode ion source and its associated electrical circuit.

\section{EXPERIMENTAL RESULTS}

In this work, all the experimental results are investigated at the optimum gap distance between the anode and the cathode, $d_{A-C}=10 \mathrm{~mm}$, with optimum inner diameter of two confinement rings equal to $7 \mathrm{~mm}$ which have been obtained before [10]. The input discharge and output ion beam characteristics of the ion source are measured using nitrogen and argon gases. Also the parameters which affect ion beam emerge from the ion source such as the distance between the ion exit aperture of the cathode and the ion collector plate and the ion source aspect ratio are determined.

\section{1 - Input discharge characteristics using nitrogen and argon gases}

Figures (2-3) show the relation between discharge current, $I_{d}$, and discharge voltage, $V_{d}$, for $d_{A-C}=10 \mathrm{~mm}$ with optimum inner diameter of two confinement rings at different pressures, P, using nitrogen and argon gases. It is clear that an increase of the discharge voltage was accompanied by an increase of the discharge current, where at the same discharge voltage; the discharge current is higher at high pressure than that at low pressure. Also at the same operating conditions such as discharge current and pressure, the discharge voltage in case of nitrogen gas start at low value than that in case of argon gas. This is due to the higher ionization potential of argon gas than that of nitrogen gas.

\section{2 - Output ion beam characteristics using nitrogen and argon gases}

Figures (4-5) show the relation between the output ion beam current, $I_{b}$, and the discharge current, $I_{d}$, for $d_{A-C}=10$

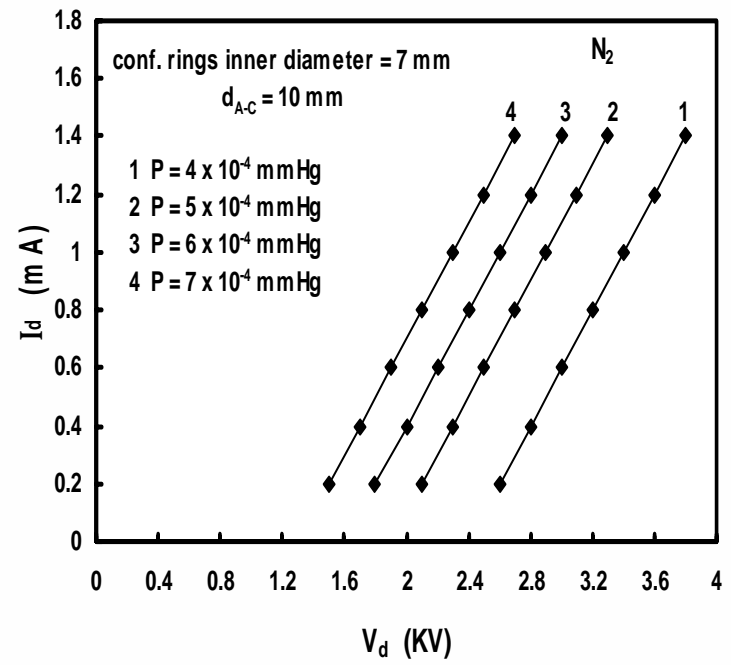

FIG. 2: Discharge current versus discharge voltage at $d_{A-C}=10 \mathrm{~mm}$ for two confinement rings of inner diameter equal to $7 \mathrm{~mm}$ using nitrogen gas.

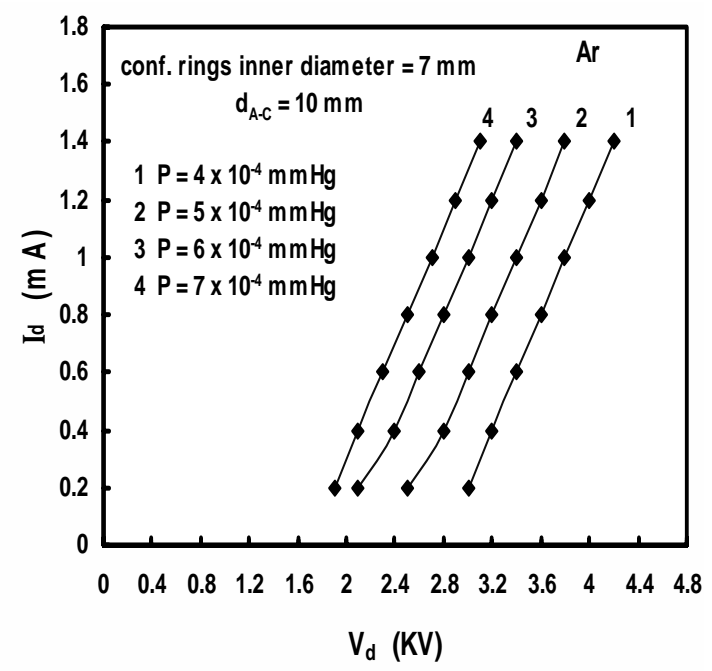

FIG. 3: Discharge current versus discharge voltage at $d_{A-C}=10 \mathrm{~mm}$ for two confinement rings of inner diameter equal to $7 \mathrm{~mm}$ using argon gas.

$\mathrm{mm}$ with optimum inner diameter of two confinement rings, the distance between the ion exit aperture of the cathode and the ion collector plate, $d_{C-C P}$, equals $4 \mathrm{~cm}$ and at different pressures using nitrogen and argon gases. It is clear from the curves that the output ion beam current increases by increasing the discharge current and at $P=4 \times 10^{-4} \mathrm{mmHg}$ and $I_{d}=1.4 \mathrm{~mA}$, a maximum output ion beam current reach $325 \mu \mathrm{A}$ and $220 \mu \mathrm{A}$ can be obtained using nitrogen and argon gases respectively. 


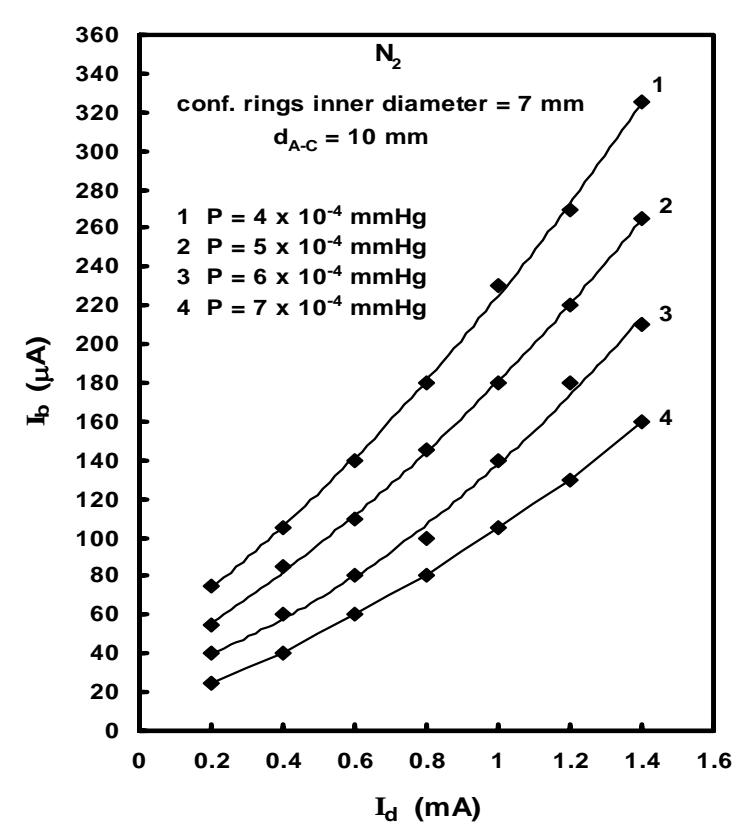

FIG. 4: Output ion beam current versus discharge current for $d_{A-C}=10 \mathrm{~mm}$ with two confinement rings of inner diameter equal to $7 \mathrm{~mm}$ at different pressures using nitrogen gas.

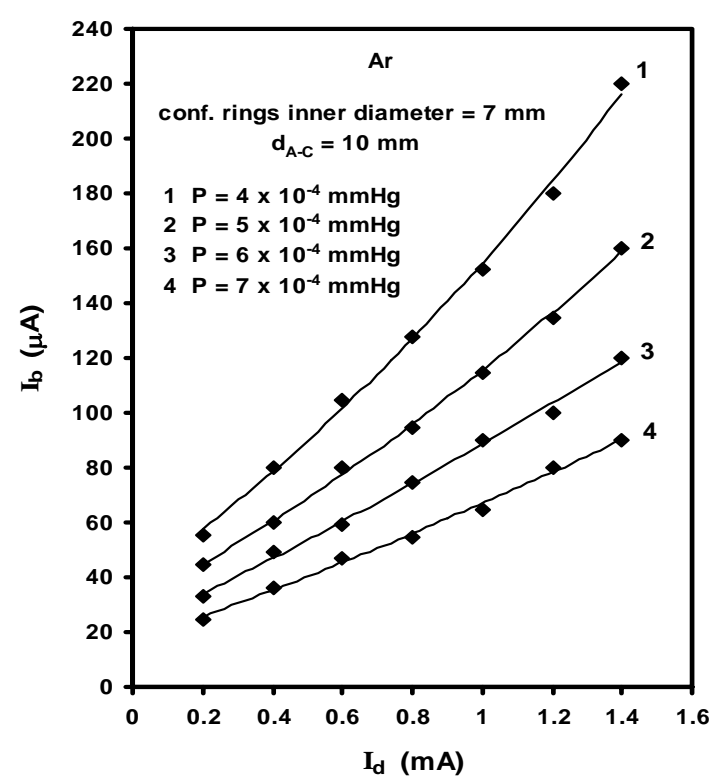

FIG. 5: Output ion beam current versus discharge current for $d_{A-C}=10 \mathrm{~mm}$ with two confinement rings of inner diameter equal to $7 \mathrm{~mm}$ at different pressures using argon gas.

\section{3 - Determination the optimum distance between ion exit aperture of the cathode - ion collector plate and the ion source efficiency}

One of the parameter which play an important role in the performance and efficiency of the ion source is adjust the distance between the ion exit aperture of the cathode and the ion collector plate, $d_{C-C p}$, for obtaining maximum output ion beam current with small divergence angle. In this work the ion collector plate has been placed at different distances from the ion exit aperture of the cathode equal to $2,3,4,4.5,5$, 5.5 and $6 \mathrm{~cm}$ respectively and at each distance the ion beam diameter obtained on the collector plate has been measured.

Figures (6 and 7) show the relation between output ion beam current versus discharge current at constant pressure for different distances between ion exit aperture of the cathode and ion collector plate using nitrogen and argon gases. It is clear that the characteristics show a family of straight line relations, where increasing the discharge current leads to increase the output ion beam current. In case of nitrogen gas at constant discharge current equals $1.2 \mathrm{~mA}$, the value of the output ion beam current reach $340 \mu A$ at $d_{C-C P}=5$ $\mathrm{cm}$ while at $d_{C-C P}=2 \mathrm{~cm}$ the output ion beam current is $140 \mu \mathrm{A}$. Also in case of argon gas at constant discharge current equals $1.2 \mathrm{~mA}$, the value of the output ion beam current reach $255 \mu A$ at $d_{C-C P}=5 \mathrm{~cm}$ while at $d_{C-C P}=2 \mathrm{~cm}$ the output ion beam current equals $100 \mu A$.

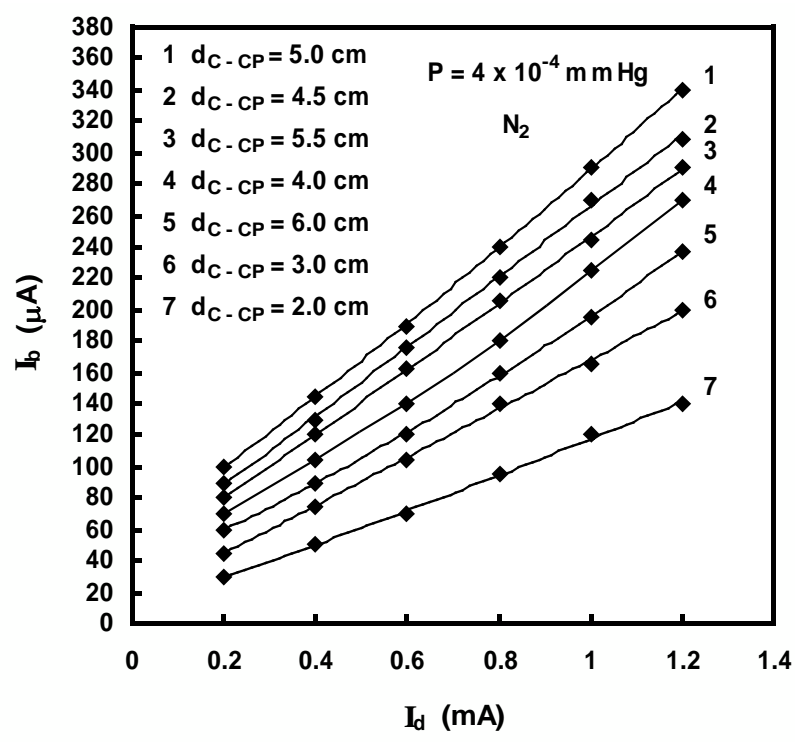

FIG. 6: Output ion beam current versus discharge current at different cathode - collector plate distances using nitrogen gas.

Fig. (8) shows output ion beam current, $I_{b}$, versus $d_{C-C P}$, at $d_{A-C}=10 \mathrm{~mm}$ with two confinement rings of inner diameter equals $7 \mathrm{~mm}$, constant pressure and discharge current using nitrogen and argon gases. It is obvious from the curves that at $d_{C-C P}=5 \mathrm{~cm}$, a maximum output ion beam current can be obtained. While before and after this optimum distance the output ion beam current decreases. Fig. (9) shows the ion source efficiency, $I_{b} / I_{d}$, versus $d_{C-C P}$, at $d_{A-C}=10 \mathrm{~mm}$ with two confinement rings of inner diameter equals $7 \mathrm{~mm}$, constant pressure and discharge current using nitrogen and argon gases. It is clear that at $d_{C-C P}=5 \mathrm{~cm}$, the ion source efficiency reaches $28.3 \%$ and $21.3 \%$ using nitrogen and argon gases respectively. But before and after the optimum distance the ion source efficiency decreases. 


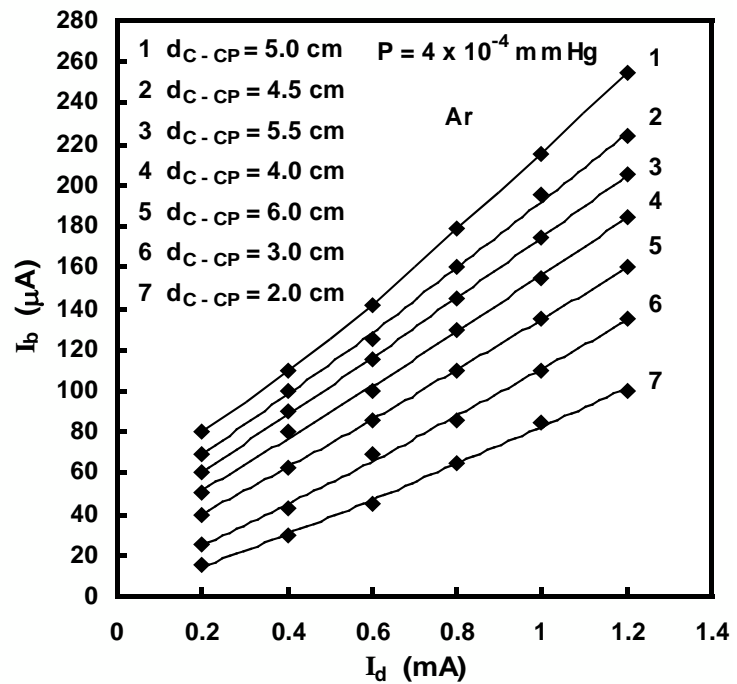

FIG. 7: Output ion beam current versus discharge current at different cathode - collector plate distances using argon gas.

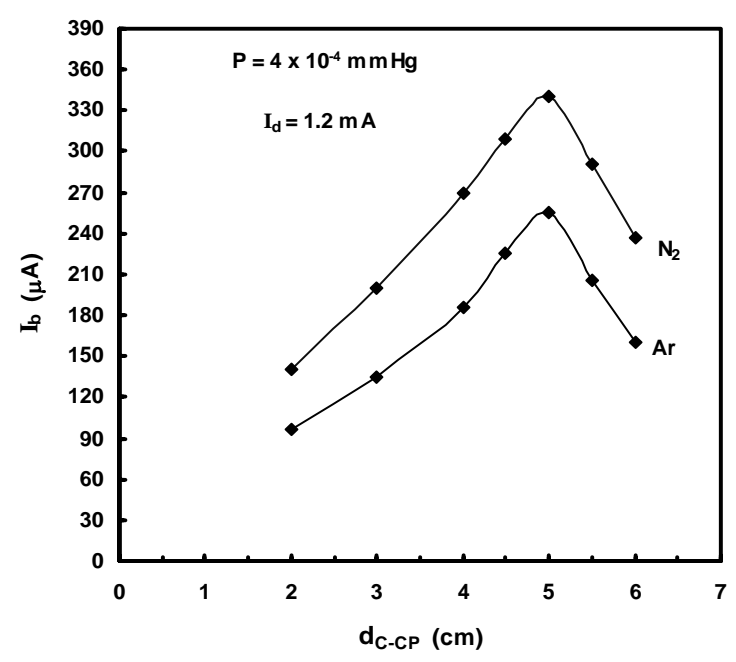

FIG. 8: Output ion beam current versus $d_{C-C P}$ using nitrogen and argon gases.

\section{4 - Determination the ion beam divergence angle}

Fig. (10) shows ion beam diameter which has been obtained on the collector plate versus $d_{C-C P}$ at constant pressure and discharge current using nitrogen and argon gases. From this figure it is clear that the ion beam diameter which obtained on the collector plate has a minimum value at $d_{C-C P}=5 \mathrm{~cm}$. While before and after this distance the ion beam diameter increases.

The divergence angle of the ion beam exit from the cath-

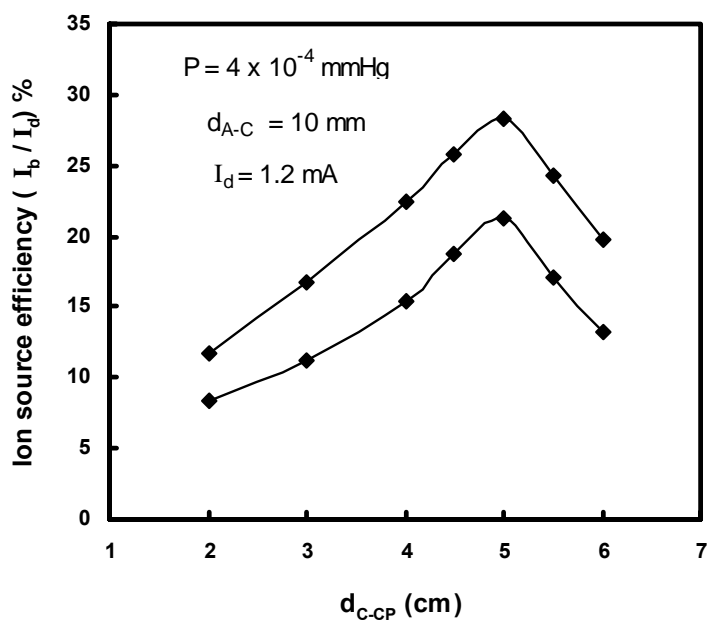

FIG. 9: Ion source efficiency versus $d_{C-C P}$ using nitrogen and argon gases.

ode aperture can be calculated using the relation:

$$
\frac{\theta}{2}=\left(\frac{r_{\text {ion beam }}-r_{\text {cathode }}}{d_{C-C P}}\right)
$$

where $\theta$ is the ion beam divergence angle, $r_{\text {ion beam }}$ is the radius of the ion beam which has been obtained on the collector plate, $r_{\text {cathode }}$ is radius of ion exit aperture of the cathode and $d_{C-C P}$ is the distance between the ion exit aperture of the cathode and the ion collector plate.

Fig. (11) shows ion beam divergence angle versus $d_{C-C P}$ at $d_{A-C}=10 \mathrm{~mm}$ with two confinement rings of inner diameter equals $7 \mathrm{~mm}$, constant pressure and discharge current using nitrogen and argon gases. From this figure it is obvious that the ion beam divergence angle decreases by increasing $d_{C-C P}$ and reach a minimum value equals $1.14^{\circ}$ and $2.29^{0}$ for nitrogen and argon gases respectively at $d_{C-C P}=5 \mathrm{~cm}$ then stat increases.

\section{5 - Determination the aspect ratio of the ion source}

The aspect ratio of the ion source has been determined from the experimental results such as discharge current and output ion beam current at different distances between ion exit aperture of the cathode and ion collector plate using nitrogen and argon gases.

Fig. (12) shows the relation between output ion beam current and aspect ratio, $\mathrm{S}$, at constant pressure and discharge current using nitrogen and argon gases. It is clear from the curves that, the maximum value of the output ion beam current is obtained at $S=0.02$ and reach $340 \mu \mathrm{A}$ and $255 \mu \mathrm{A}$ for nitrogen and argon gases respectively. This indicates that the optimum $d_{C-C P}=5 \mathrm{~cm}$.

\section{CONCLUSION}

From the experimental results of this work, it can be concluded that at $P=4 \times 10^{-4} \mathrm{mmHg}, I_{d}=1.4 \mathrm{~mA}$ and 


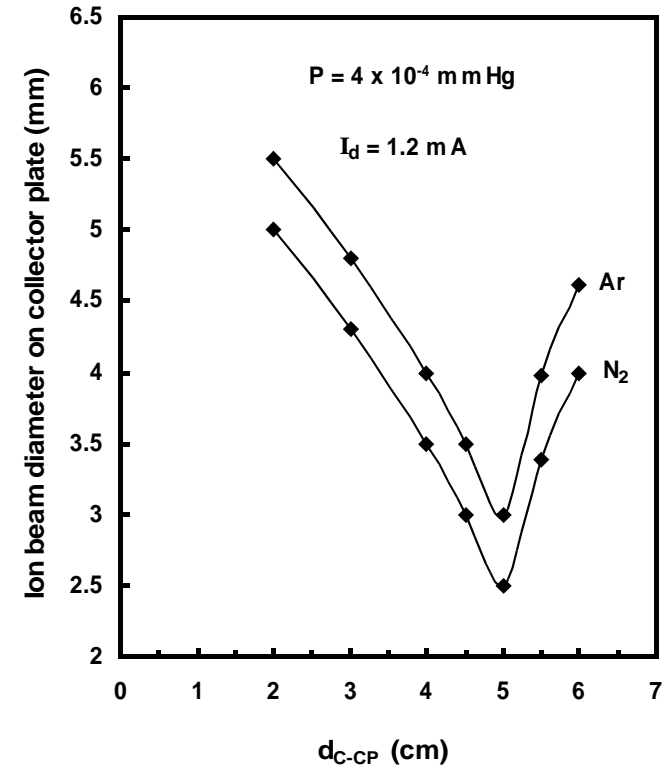

FIG. 10: Ion beam diameter on the collector plate versus distance between the ion exit aperture of the cathode and the ion collector plate using nitrogen and argon gases.

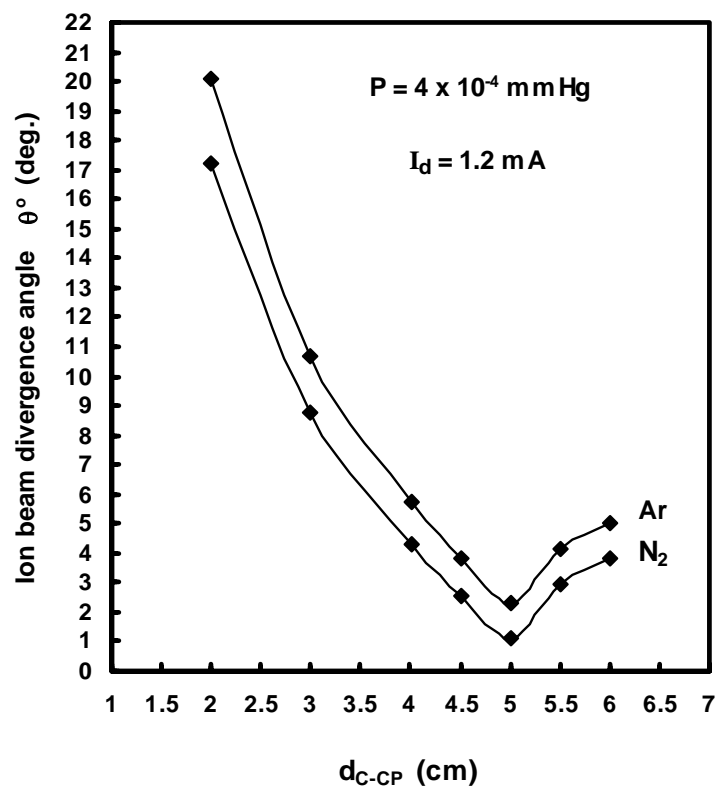

FIG. 11: Ion beam divergence angle versus the distance between the ion exit aperture of the cathode and the ion collector plate using nitrogen and argon gases.
$d_{C-C P}=4 \mathrm{~cm}$, a maximum output ion beam current reach $325 \mu A$ and $220 \mu A$ can be obtained using nitrogen and argon gases. But from the optimum distance curves between ion exit aperture of the cathode and ion collector plate, it is found that at $d_{C-C P}=5 \mathrm{~cm}, P=4 \times 10^{-4} \mathrm{mmHg}$ and $I_{d}=1.2 \mathrm{~mA}$, a maximum output ion beam current reach $340 \mu \mathrm{A}$ and $255 \mu \mathrm{A}$ can be obtained using nitrogen and argon gases. The ion source efficiency reached $28.3 \%$ and $21.3 \%$ using nitrogen and argon gases respectively. Therefore, it can be concluded that a maximum output ion beam current can be obtained at $d_{C-C P}=5 \mathrm{~cm}$. Also at this optimum distance, a minimum value of ion beam divergence angle reach $1.14^{\circ}$ and $2.29^{\circ}$ can be obtained using nitrogen and argon gases. It can be concluded that a maximum output ion beam current can be obtained at $S=.02$, i.e at $d_{C-C P}=5 \mathrm{~cm}$. Therefore for exposure any specimen to maximum ion beam current with small divergence angle, the specimen must be placed at a distance equals $5 \mathrm{~cm}$ from the ion exit aperture of the cathode. This ion source is suitable for ion beam applications such as, sputtering, etching, material surface modification and micromachining.

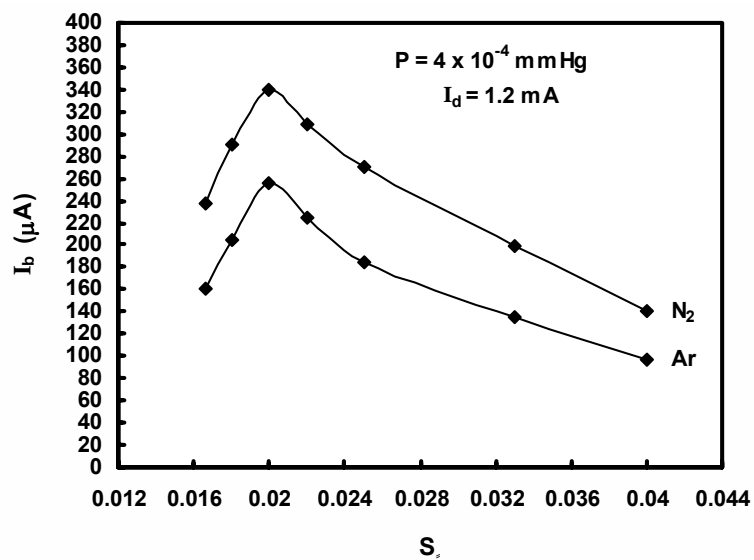

FIG. 12: Output ion beam current versus aspect ratio at constant pressure and discharge current using nitrogen and argon gases.
[1] L.A. Giannuzzi and F.A.Stevie; "Introduction to Focused Ion Beams", Springer, USA (2005).

[2] S.I. Molokovsky and A.D. Sushkov; "Intense Electron and Ion Beams", Springer, Germany (2005).

[3] B.H. Wolf, "Handbook of Ion Sources", CRC Press, Boca Ra- ton, New York (1995).

[4] I.A. Lieberman and L.J. Lichtenberg; "Principles of Plasma Discharges and Materials Processing", John Wiley \& Sons, NJ, USA (2005)

[5] I.G. Brown; " The Physics and Technology of Ion Sources," 
Wiley-VCH Verlag GmbH \& Co. KGaA, Weinheim, Germany (2004).

[6] R. Hellborg; "Electrostatic Accelerators", Springer, Netherlands (2005).

[7] R. Behrisch and W. Eckstein; "Sputtering by Particle Bombardment", Springer, Berlin (2007).

[8] R.J. Rodrguez, A. Medrano, J.A. Garca, G.G. Fuentes, R. Martínez and J.A. Puertolas; Surface and Coatings Technol- ogy, 201(19-20), 8146 (2007).

[9] T. Sadahiro, K. Matsuyama, M. Kiuchi, T. Matumoto, T. Takizawa, S.Sugimoto, T. Fukuda and S.Goto; Surface and Coatings Technology; 188, 265 (2004).

[10] A.G. Helal, S.A. Nouh, H. El-Khabeary and S.M. Mahmoud; Arab J. Nucl. Sci. Appl.; Accepted for Publication Feb. (2010). 